Why it is so hard to predict our partner's product preferences: The effect of target familiarity on prediction accuracy

Davy Lerouge and Luk Warlop

DEPARTMENT OF MARKETING AND ORGANISATION STUDIES (MO) 


\title{
Why It Is So Hard to Predict Our Partner's Product Preferences: The Effect of Target Familiarity on Prediction Accuracy
}

\author{
DAVY LEROUGE \\ LUK WARLOP*
}

\begin{abstract}
Many buying decisions require predictions of another person's product attitudes. Yet, consumers are often inaccurate predictors, even for familiar others. We provide strong evidence that target familiarity can even hurt accuracy in the presence of attitude feedback. Although overprojection and lack of product-specific attitude information have been identified as possible reasons for prediction inaccuracy, our results suggest a retrieval explanation. When presented with product-specific attitude feedback, predictors adapted their level of projection and encoded the attitude information, but they did not use this information. Instead, they retrieved less diagnostic, pre-stored information about the familiar targets to predict their product attitudes.
\end{abstract}

$\mathrm{F}_{\mathrm{p}}^{\mathrm{o}}$ or many product decisions consumers predict others' product attitudes. For instance, consumers are found to make predictions about their co-consumers' product preferences before engaging in a joint decision process, such as buying furniture or deciding where to have dinner (e.g., Krishnamurthi 1983; Park 1982). Also when buying products for others, such as when purchasing gifts, consumers search for a product that matches the receiver's attitudes (e.g., Wooten 2000). Research on agent decision making provides additional examples of people, varying from knowledgeable neighbors to trained experts (like financial analysts and real estate agents), who make product attitude predictions for others (e.g., Solomon 1986; West 1996). Also in personal selling, good salespeople need to empathize with the complex feelings and preferences of their customers (Weitz, Sujan, and Sujan 1986). Similarly, product managers often rely on their own intuitive predictions about consumers

*Davy Lerouge is assistant professor of marketing at Tilburg University, P.O. Box 90153, 5000 LE Tilburg, the Netherlands (d.lerouge@uvt.nl). Luk Warlop is professor of marketing at the Katholieke Universiteit Leuven, Naamsestraat 69, 3000 Leuven, Belgium (luk.warlop@econ.kuleuven .ac.be). This article is based on the first author's doctoral dissertation. The authors acknowledge the helpful input of the editor, the associate editor, and reviewers. In addition, the authors thank Joe Alba, Marnik Dekimpe, Siegfried Dewitte, Steve Hoch, Mario Pandelaere, Rik Pieters, Dirk Smeesters, and Piet Vanden Abeele for their valuable comments on an earlier version of this article.

Dawn Iacobucci served as editor and Eric Arnould served as associate editor for this article.

Electronically published November 14, 2006
(Hoch 1988). If not, they use marketing research tools that are frequently based on proxy reports by key informants who are asked to predict the product feelings of others (Menon et al. 1995). Finally, even for many decisions that are made individually and for their own use, consumers value social approval. For example, people take into account the attitudes and preferences of referents such as coworkers and friends when buying luxury and public products (e.g., Childers and Rao 1992). People who really like a specific brand or product will hesitate to buy it if they predict that their peers will not share their preference. In sum, consumers (and marketers) predict the other's product attitudes in many different situations. Hence, it is important to obtain more insight into factors that affect the accuracy of these product predictions.

In this article, we directly test the effect of familiarity with the target person (i.e., for whom one is predicting the product attitudes) on prediction accuracy. Although it could intuitively be expected that target familiarity results in more accurate predictions due to the extensive amount of prestored target information, there is already some evidence that people fail to properly predict a familiar target's product attitudes. Marketing experts, for instance, were no more accurate than novices in predicting the opinions of the U.S. consumer (Hoch 1988). Of course, predicting the product attitudes of a group differs from predicting the attitudes of an individual. Yet, inaccuracy has also been observed when consumers predict the product attitudes of familiar individuals, like relationship partners. Davis, Hoch, and Ragsdale (1986) found that only $53 \%$ of the partners were able to outperform a hypothetical forecaster who simply used the 
average gender-specific preference. Related research in social psychology consistently demonstrated that neither relationship length nor relationship involvement with the target person necessarily increases accuracy in predicting beliefs, and personality traits (e.g., Swann and Gill 1997). Kenny and Acitelli (2001), for instance, surprisingly found that partner-related information contributed less to the overall prediction accuracy than the predictor's own attitudes did. However, these low levels of accuracy have typically been observed for predictions on which diagnostic information about the familiar targets is rather sparse. Davis et al. (1986) used new (at that time) futuristic products, such as a pop-top can for canned vegetables. Kenny and Acitelli (2001) observed only low contributions of partner information for predictions central to the relationship (e.g., feelings of closeness, sexual enjoyment) and, therefore, probably quite sensitive, personal, and hardly discussed with the partner. The opposite was found for topics less central to the relationship (job satisfaction and feelings about the partner's family), on which partners presumably have exchanged more information. Thus, a lack of diagnostic information about the familiar target's product-specific attitudes seems an obvious and acceptable explanation why target familiarity so far has been found to seldom affect the accuracy of product predictions. This explanation also implies that it would be easy to overcome prediction inaccuracy, once diagnostic target information is made available. In fact, West's (1996) finding that people quickly learn to predict a complete stranger's product attitudes when provided with product-specific attitude feedback comports nicely with this reasoning.

The main contribution of the present study is to provide convincing evidence that target familiarity induces prediction problems far beyond a simple lack of diagnostic information. In fact, our main hypothesis is that familiarity with the target person will even have a negative effect on prediction accuracy in the presence of product-specific target feedback. Predictors can use this diagnostic feedback, but they can also put a heavy weight on their own product attitudes or on a variety of pre-stored information about the target (e.g., Davis et al. 1986; Kenny and Acitelli 2001). Hence, we distinguish two possible explanations for the predicted negative effect of target familiarity on prediction accuracy. First, it can be that predictors overproject their own attitudes on familiar others despite the availability of product-specific feedback. Although West (1996) found that predictors of a stranger's product attitudes were able to adapt their level of projection based on product-specific attitude feedback, we expect that predictors will stick to their own attitudes when this feedback belongs to a familiar target. For familiar targets, attitude similarity assumptions appear to be much stronger than for unfamiliar ones (e.g., Moreland and Zajonc 1982). Aron et al. (1991) even argued that information about familiar others is mixed with information about the self. So it can be expected that predictors, at least initially, assume the familiar other's product attitudes to be quite similar. These high similarity expectations likely color the interpretation of new but ambiguous attitude information (e.g., Stapel and Schwarz 1998) and motivate predictors to ignore attitudinal discrepancies (e.g., Davis and Rusbult 2001). Consequently, high levels of own attitude projection may persist, despite the availability of veridical feedback about the target's product attitudes. Second, the predicted negative effect of familiarity may also be the result of an overly high reliance on pre-stored target information. The information that people possess about familiar others has often been found to be invalid or irrelevant when predicting specific product attitudes (e.g., Davis et al. 1986; Swann and Gill 1997). Despite its low validity, predictors tend to use pre-stored information, thereby insufficiently adjusting for more diagnostic feedback (e.g., Broniarczyk and Alba 1994). Such overweighing of pre-stored target information will very likely increase with target familiarity. Predictors typically possess an elaborate knowledge base about familiar others. This huge diversity of pre-stored information about familiar targets may cause predictors to have high confidence in their prediction abilities (Swann and Gill 1997) and, in turn, limit their attention and sensitivity to new, product-specific attitude feedback (Alba and Hutchinson 2000). Moreover, even if the product-specific attitude information is encoded properly, the impact of this truly diagnostic information is likely to be diluted by the extensive amount of irrelevant information about the familiar target (e.g., Nisbett, Zukier, and Lemley 1981; Tetlock and Boettger 1989).

Taking these two explanations together, we predict that target familiarity will have a negative effect on prediction accuracy if product-specific attitude feedback is available because people will put heavy weight on their own attitudes and their pre-stored target information at the cost of more diagnostic attitude feedback. To test the specific effect of target familiarity on the prediction process, we designed three experiments in which we asked participants to predict their relationship partner's attitudes toward a series of product alternatives. Instead of manipulating the prediction target, we manipulated the awareness that one is predicting the partner's attitudes. More specifically, in some conditions we disguised the true identity of the target by telling the participants they had to predict the product attitudes of a stranger. In other conditions, we revealed the true identity of the target and asked the participants to predict their partner's product attitudes. By keeping the target (i.e., the partner) constant in all conditions, we avoided confounds (like differences in attitude similarity). This way, we were able to test the effect of target familiarity on a variety of prediction measures. To calculate these measures we used the established framework proposed by Hoch $(1987,1988$; see the appendix for more details). First, prediction accuracy can be measured as the correlation between the predictions and the target person's actual attitudes. This overall accuracy measure can be decomposed into the component due to the projection of own product attitudes and the component due to all other information beyond own attitudes (i.e., pre-stored target information and product-specific attitude feedback). 
The accuracy-by-own attitudes can be measured as the level of projection multiplied by the actual similarity of the partner's product attitudes. Intuitively, this means that when partners have highly similar attitudes, predictors will attain high levels of accuracy by projecting their own attitudes. However, when the predictor's attitudes and those of the target person are uncorrelated (i.e., attitude similarity is close to zero), projection will not add much to the overall prediction accuracy. In turn, the level of projection (or the weight given to own attitudes) can be measured as the correlation between own attitudes and the attitude prediction. The accuracy-by-other information is the overall prediction accuracy minus the accuracy-by-own attitudes. Applying these measures in a series of experiments, it is consistently found that target familiarity has a negative effect on prediction accuracy when attitude similarity is low. Additionally, the first experiment shows that target familiarity does not affect the level of projection or accuracy-by-own attitudes. Instead, familiarity is found to have a negative effect on accuracy-by-other information. A second experiment provides evidence that this negative familiarity effect on other information was mainly caused by an overweighing of pre-stored target information at the cost of more relevant product-specific attitude feedback of their partner. The third experiment further demonstrates that, although predictors properly encoded the attitude feedback, they did not fully use this information during subsequent product predictions.

\section{EXPERIMENT 1}

\section{Stimuli, Participants, and Design}

We compiled a heterogeneous set of 30 different bedroom images from furniture store catalogs, interior decoration magazines, and internet sites to serve as product alternatives. Each product alternative consisted of a picture of the bedroom, the brand name (e.g., Vannes, Cerenna, and so forth), a description of the set-up (e.g., bed, two bedside tables and dresser), the finishing (e.g., cherry, rattan, and so on), and the price. The stimuli were scanned from catalogs or downloaded from the Internet to be presented on a color computer monitor.

Thirty-five couples who were in a relationship for at least 6 mo. (average 28 mo.) were invited to the laboratory. Participants were seated in separate, individual cubicles equipped with a computer on which the experiment was conducted. During the entire session, the participants had no contact with their partner or other participants. First, they provided their own attitudes toward the 30 different furniture sets (by choosing "positive" or "negative"). Next, half of the participants were asked to predict their partner's attitudes (positive or negative) toward those furniture sets. After each prediction, the partner's actual attitude (positive or negative) was presented. The other participants followed the same procedure, but instead of their partner's furniture attitudes, they were asked to predict the attitudes of a stranger, called person X. However, in reality, this person $\mathrm{X}$ was also their partner. So, the relationship partner was the target's true identity for all participants, but for half of them this was disguised.

We also included similarity in our design. Similarity was calculated as the correlation between the predictor's and the partner's actual product attitudes, on which a median split was performed (median $=.19$ ). This resulted in a 2 (familiarity: disguised vs. revealed) $\times 2$ (similarity: low vs. high similars) between-subjects design.

\section{Results}

The reported results were obtained by analyzing each participant as an individual observation. As suggested by the reviewers, we reanalyzed our data on two subsets that included just one member of each couple to account for potential interdependence between the observations. The results on both subsets replicated the findings that are reported in the current and subsequent experiments and are not further documented.

Our main hypothesis was that target familiarity would negatively affect prediction accuracy. Next to a positive effect of similarity on accuracy $\left(M_{\text {low }}=.14, M_{\text {high }}=.42\right.$; $F(1,66)=40.70, p<.0001)$, the results partially confirmed our hypothesis. People who predicted their partner's attitudes $(M=.24)$ were less accurate than those for whom target familiarity was disguised $(M=.36 ; F(1,66)=$ $3.08, p<.09)$. However, the effect of familiarity was qualified by similarity $(F(1,66)=4.50, p<.04$; panel A of fig. 1). Post hoc analyses showed that low similars predicted the product attitudes less accurately when the target's true identity was revealed $(M=.05)$ compared to when it was disguised $(M=.22)$. For high similars, no effect of target familiarity was observed $\left(M_{\mathrm{disg}}=.41, M_{\mathrm{rev}}=.43\right)$. To obtain an explanation for these observed effects, we performed similar analysis on the other prediction measures.

First, target familiarity did not influence the accuracy-byown attitudes component $(F(1,66)=.62, p>.43$; panel $\mathrm{B}$ of fig. 1). The level of accuracy that the participants obtained by projecting their own product attitudes was not significantly different in the revealed condition $(M=.12)$ compared to the disguised condition $(M=.14)$. We found only that high similars $(M=.26)$, unsurprisingly, obtained a higher level of accuracy-by-own attitudes than low similars $(M=.00 ; F(1,66)=84.29, p<.0001)$. More interestingly, we observed a significant familiarity by similarity interaction effect on the accuracy-by-other information component $(F(1,66)=11.04, p<.01$; panel $\mathrm{C}$ of fig. 1$)$. Post hoc analyses revealed that, for low similars, the accuracyby-other information was lower when the target's identity was revealed $(M=.05)$ compared to when it was disguised $(M=.23)$. For high similars, this negative familiarity effect was not observed $\left(M_{\text {disg }}=.13, M_{\text {rev }}=.20\right)$.

One possible explanation for these findings is that low similars overestimated the similarity with their partner and heavily weighted their own attitudes, thereby reducing the impact of other information on prediction accuracy. Yet, the results on the level of projection showed that participants did not project significantly more when target familiarity 
FIGURE 1

EXPERIMENT 1: DECOMPOSITION OF THE INTERACTION BETWEEN FAMILIARITY AND SIMILARITY

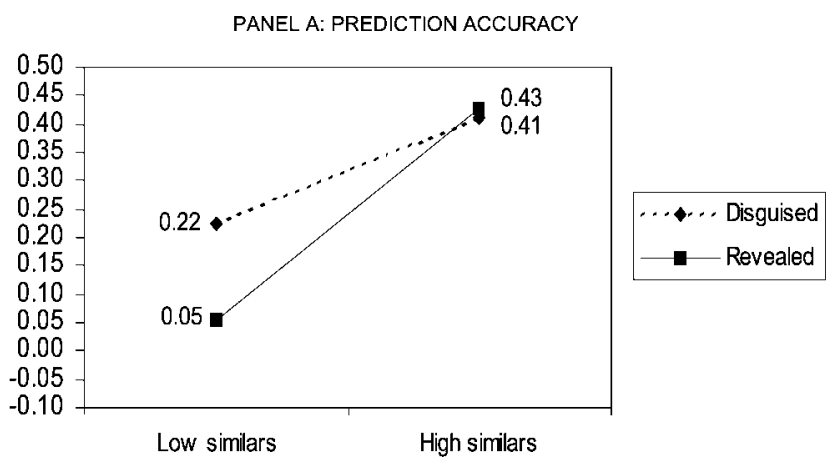

PANEL B: ACCURACY-BY-OWN ATTITUDES

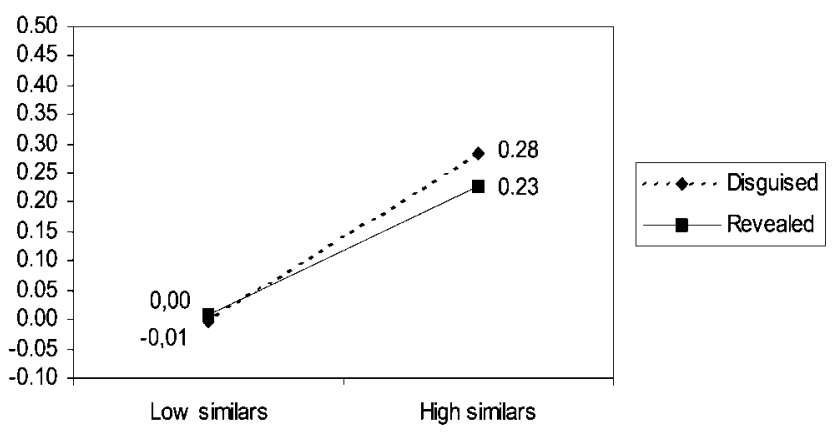

PANEL C: ACCURACY-BY-OTHER INFORMATION

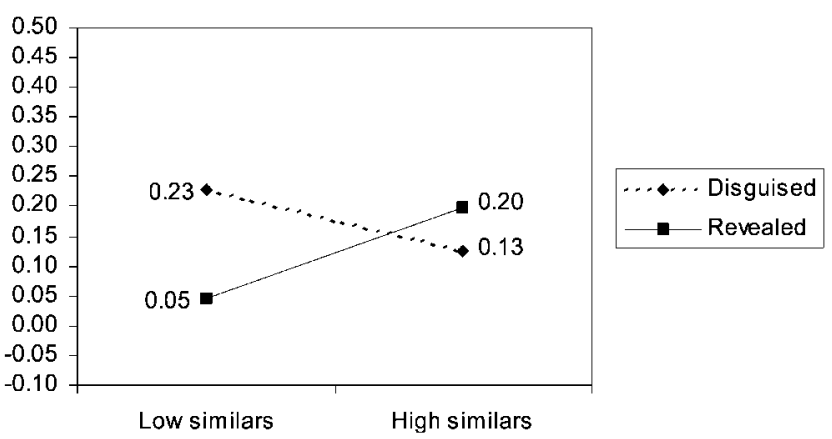

was revealed $(M=.45)$ than when it was disguised $(M=.42 ; F(1,66)=.27, p>.60)$. Instead, we found only that high similars $(M=.56)$ projected more than low similars $(M=.32 ; F(1,66)=18.10, p<.0001)$. Thus, the participants adapted their level of projection to their actual level of attitude similarity, independently of their familiarity with the target.

\section{Discussion}

This study provides support for our main hypothesis. In the presence of product-specific attitude information, target familiarity can cause prediction inaccuracy. This was observed only when similarity on furniture attitudes was low and, therefore, own attitudes are a nondiagnostic cue for the attitude predictions. The simplest explanation is that consumers, when predicting the product attitudes of familiar but dissimilar others, continue to rely heavily on their own product attitudes at the cost of more diagnostic, other information. Yet, the results on the amount of projection are inconsistent with this overprojection explanation. Previous studies that observed higher levels of projection among familiars typically focused on sensitive issues that are more central to the relationship (e.g., Davis and Rusbult 2001). It is quite likely that the partner's furniture attitudes are not perceived as critical to the relationship and, therefore, no overprojection occurred. A second explanation is that target familiarity negatively affects the validity of own attitudes when similarity is low. It could be that predictors, who receive attitude feedback about their partner that is incongruent with the self, elicit thoughts about their own product attitudes resulting in unstable own attitudes (Wilson and Schooler 1991). However, besides not affecting the weight of own attitudes, target familiarity also had no effect on the accuracy component caused by own attitudes. Thus, we can conclude that neither differences in weight nor in validity can explain the observed negative effect of target familiarity on prediction accuracy.

What is causing the accuracy differences then? The overall accuracy difference is entirely reflected in the accuracyby-other information component. When similarity is low, predictors are found to rely heavily on other information beyond own attitudes, and this is where it goes wrong. More specifically, our findings suggest that the predictive quality of the other information about the partner is significantly lower than that about a stranger. Since this other information consists of both pre-stored target information and the product-specific feedback, this implies that target familiarity must negatively affect the validity or weight of these two types of information. Given that the validity of the productspecific feedback (i.e., the partner's actual product attitudes) is independent of our familiarity manipulation, one of the remaining explanations of our findings is that the pre-stored information about a familiar target is less valid than that of a stranger. People predicting the attitudes of an unfamiliar target can rely only on general and stereotypical information about the stranger, which may be quite diagnostic (Nisbett and Kunda 1985). When predicting familiar targets, they are likely to ignore this valid information. Instead, they use more specific target information that is often found to be invalid or irrelevant when predicting product attitudes (e.g., Davis et al. 1986; Swann and Gill 1997). To test this explanation, we manipulate the availability of feedback in experiment 2. When no product-specific feedback is provided, we are able to observe the effect of target familiarity on the prediction validity of pre-stored target information only. 


\section{EXPERIMENT 2}

\author{
Stimuli, Participants, and Design
}

Eighty-four couples (average relationship length of 26 mo.) participated in this experiment. Half of the participants followed the same procedure as in experiment 1 and received the actual attitude of their partner after each prediction. The others followed a similar procedure except that they did not receive any feedback during the prediction task. The design was a 2 (familiarity: disguised vs. revealed) $\times 2$ (similarity: low vs. high similars) $\times 2$ (feedback: no feedback vs. feedback) between-subjects design.

\section{Results}

If the familiarity effect is mainly driven by differences in pre-stored target information, then we should also find an effect of target familiarity on prediction accuracy in the no feedback conditions. We observed a three-way interaction effect on prediction accuracy $(F(1,159)=3.65, p<$ .06 ; panel A of fig. 2). When the predictors received attitude feedback, as in experiment 1 , the results confirmed that the familiarity effect on accuracy depended on the level of similarity $(F(1,77)=7.76, p<.01)$. Low similars for whom target familiarity was disguised $(M=.24)$ were more accurate than those for whom it was revealed $(M=.06$; $F(1,77)=9.19, p<.01)$. For high similars, no familiarity effect was observed $\left(M_{\text {disg }}=.26, M_{\text {rev }}=.32 ; F(1,77)=\right.$ $.90, p>.34)$. Interestingly, when predictors received no feedback, the significant familiarity by similarity effect disappeared $(F(1,82)=.001, p>.98)$ and prediction accuracy was affected only by similarity $\left(M_{\text {low }}=.13, M_{\text {high }}=.29\right.$; $F(1,82)=13.20, p<.001)$. This suggests that pre-stored information about an alleged stranger is equally diagnostic as pre-stored information about a familiar target. If this is true, we should also observe no effects of familiarity on accuracy-by-other information when no feedback was provided and the other information consisted only of pre-stored target information.

While we again observed only a positive effect of similarity on accuracy-by-own attitudes $\left(M_{\text {low }}=.02, M_{\text {high }}=\right.$ $.19 ; F(1,159)=115.55, p<.0001$; panel B of fig. 2$)$, we found the expected three-way interaction on accuracy-byother information $(F(1,159)=3.77, p<.05$; panel $\mathrm{C}$ of fig. $2)$. When feedback was provided, the effect of familiarity was qualified by similarity $(F(1,77)=4.79, p<.04)$. Low similars used more valid other information when target familiarity was disguised $(M=.21)$ compared to when it was revealed $(M=.02 ; F(1,77)=12.59, p<.001)$. For high similars, this difference was not significant $\left(M_{\text {disg }}=.09\right.$, $\left.M_{\text {rev }}=.10 ; F(1,77)=.03, p>.85\right)$. Yet, in the no feedback condition, target familiarity did not significantly affect accuracy-by-other information $\left(M_{\text {disg }}=.09, \quad M_{\text {rev }}=.13\right.$; $F(1,82)=.66, p>.42)$.

The results on the level of projection further confirmed that, also in this experiment, target familiarity did not affect the weight given to own attitudes $\left(M_{\mathrm{disg}}=.38, M_{\mathrm{rev}}=\right.$
$.44 ; F(1,159)=2.18, p>.14)$. We observed only that high similars $(M=.47)$ projected more than low similars $(M=.35 ; F(1,159)=8.70, p<.01)$.

\section{Discussion}

The findings of the present experiment rule out the possibility that the pre-stored information for predicting a familiar target is lower in quality than the general information to predict an alleged stranger. This means that an overly heavy weight on pre-stored target information at the cost of product-specific attitude feedback is the only remaining explanation for the observed familiarity effects. The pre-stored information that people possess about their partner is extensive. Hence, it could be that this elaborate knowledge makes predictors overly confident, such that they even do not attend to the product-specific attitude feedback (Swann and Gill 1997). Alternatively, it could also be that predictors, even though they attend to the feedback, fail to learn because they assume that they already possess diagnostic prediction cues or because they interpret the feedback to be consistent with pre-stored beliefs and expectations (Stapel and Schwarz 1998). Third, it is possible that predictors attend to productspecific attitude feedback and also learn from it, but still retrieve some pre-stored target information. Previous studies observed that, even if available information is irrelevant, predictors try to extract useful information from it (e.g., Nisbett et al. 1981; Tetlock and Boettger 1989). In experiment 3, we investigate whether the familiarity effect was mainly caused by attention, learning, or retrieval biases by manipulating the moment that we revealed the target as being the predictor's partner and by adding a memory test.

We also wanted to investigate to what extent our findings could be generalized across learning tasks. Therefore, we added a purely observational learning task (cf. Broniarczyk and Alba 1994). This learning task is more realistic, less involving, and more limited than the prediction-learning task used in the previous experiments. In the latter, people receive information not only about the prediction target but also about the validity of their prediction strategy.

\section{EXPERIMENT 3}

\section{Stimuli, Participants, and Design}

We added more stimuli to obtain 60 bedroom furniture sets in total. One hundred and twelve couples (average acquaintance of 26 mo.) provided their own attitudes toward all bedroom sets. Next, they completed three more tasks: a learning task, a prediction task, and a memory test. During the learning task, participants either predicted and subsequently received the target's actual attitudes or they immediately observed the target's product attitudes toward a first subset of 30 bedroom sets. In the prediction task, all participants predicted their target's attitudes on the remaining 30 bedroom sets, and no feedback on the actual attitudes was provided. The two bedroom subsets were counterbalanced, and the presentation order within each subset was 
FIGURE 2

EXPERIMENT 2: DECOMPOSITION OF THE INTERACTION BETWEEN FAMILIARITY, SIMILARITY, AND FEEDBACK

PANEL A: PREDICTION ACCURACY

No Feedback

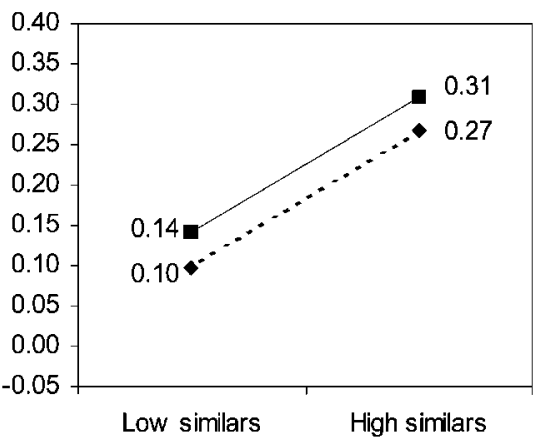

Feedback

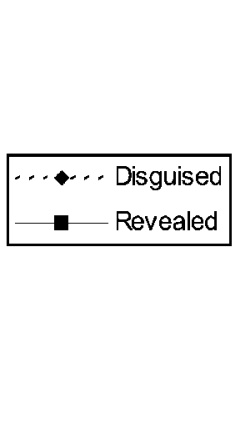

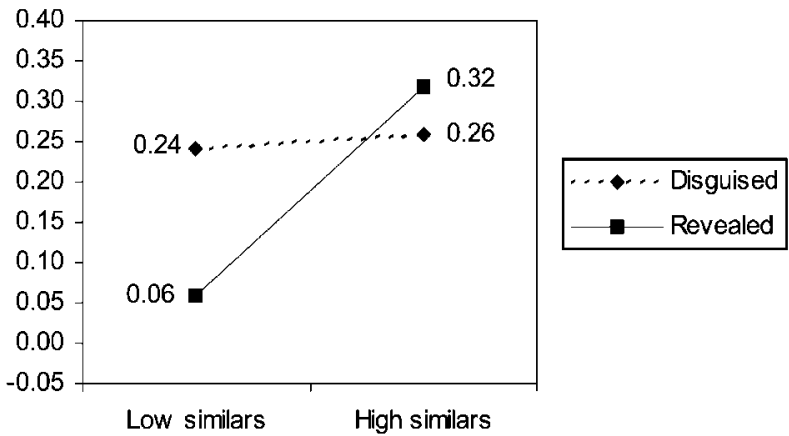

Low similars High similars

PANEL B: ACCURACY-BY-OWN ATTITUDES

No Feedback

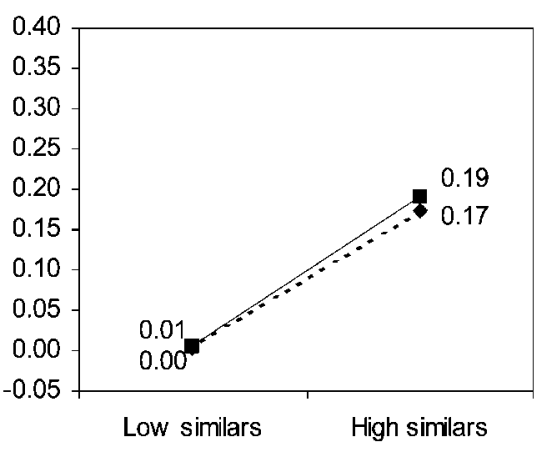

Feedback

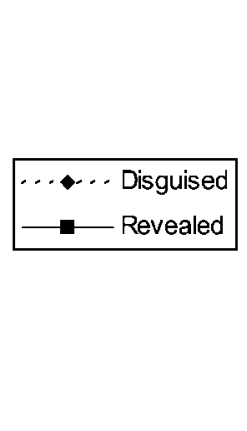

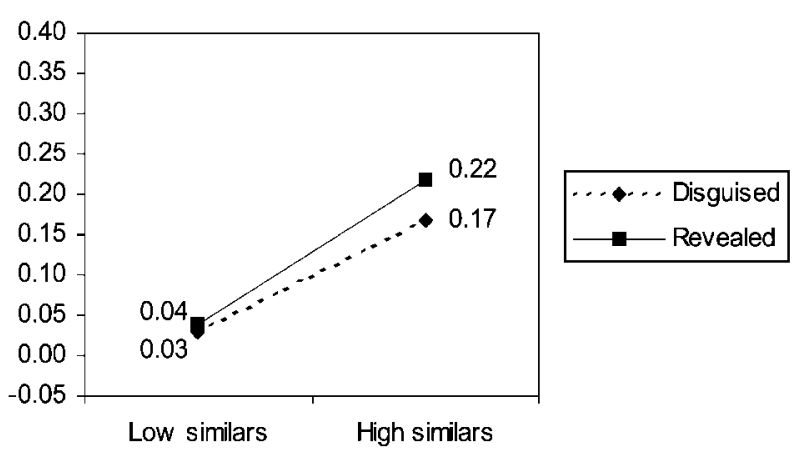

PANEL C: ACCURACY-BY-OTHER INFORMATION

No Feedback

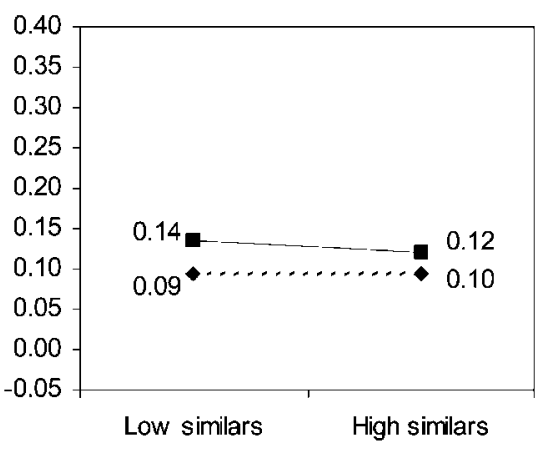

Feedback

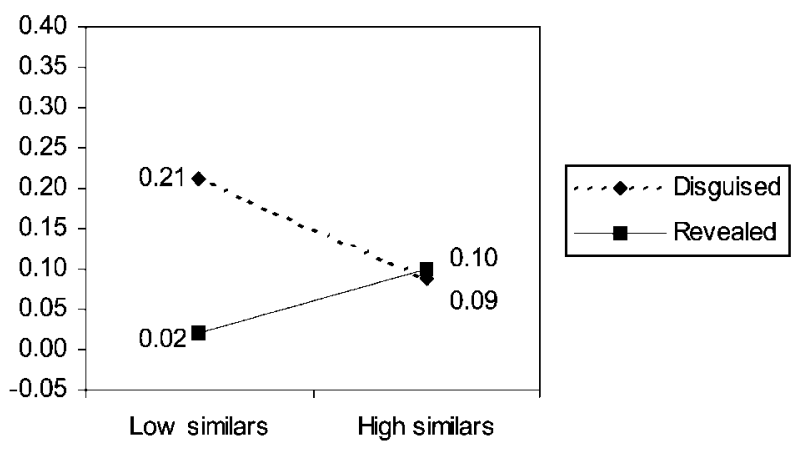


randomized. Finally, during a memory test, participants were asked to recall the target's attitudes (positive, negative, or no idea) toward 15 furniture sets that were randomly chosen from those they saw during the learning task. The computer program failed to store the answers on this memory test of one participant. This participant was excluded from the analyses that were performed on the memory test data.

Following Cohen (1981), we disentangled the familiarity effect on the encoding (i.e., attention and learning) processes from its effect on retrieval by manipulating the moment of target identification: before the learning task, after learning but before the prediction task, or not at all. For participants in the prelearning condition, target familiarity can affect encoding during the learning task as well as retrieval of attitude information during the subsequent prediction task. In the postlearning condition, familiarity may still affect the retrieval, but not encoding. Finally, target familiarity cannot influence any of these processes in the control condition where the target's identity remained disguised. In sum, this experiment had a 3 (familiarity: prelearning vs. postlearning vs. control) $\times 2$ (similarity: low vs. high similars $) \times 2$ (learning task: prediction vs. observation) between-subjects design.

\section{Results}

If differential attention was causing the familiarity effect, then participants in the prelearning condition should recall less original product attitudes during the memory test compared to those in the other conditions. Yet, no effect of target familiarity was observed $\left(M_{\text {pre }}=10.75, M_{\text {post }}=10.75\right.$, $\left.M_{\text {control }}=10.10 ; F(2,213)=10.11, p>.10\right)$. We found only that people who observed the attitudes during the learning task $(M=11.29)$ recalled more attitudes correctly than those who learned by prediction $(M=9.78 ; F(1,213)=$ 27.87, $p<.0001)$.

If familiarity affects learning from attitude feedback, then prediction accuracy in the prelearning condition should be lower than in the postlearning condition. As expected, the main effect of target familiarity on prediction accuracy was significant $(F(2,214)=5.89, p<.01$; panel $A$ of fig. 3$)$. However, no difference was observed between the prelearning $(M=.19)$ and postlearning condition $(M=.22$; $F(1,214)=1.03, p>.31)$. Instead, participants in the prelearning and postlearning conditions $(M=.21)$ were less accurate compared to those in the control condition $(M=$ $.30 ; F(1,214)=10.81, p<.01)$. This is inconsistent with the learning explanation but in line with the retrieval explanation. Although target familiarity did not bias the attention paid to and learning from the attitude feedback during the learning task, it induced retrieval deficiencies during the subsequent prediction task. These effects were found independent of the learning task $(F(1,214)=1.03, p>$ .35). Additionally we observed that participants who learned the target's furniture attitudes by prediction $(M=.26)$ tended to be more accurate than those who learned by observation $(M=.21 ; F(1,214)=3.75, p<.06)$ and that
FIGURE 3

\section{EXPERIMENT 3: DECOMPOSITION OF THE MAIN EFFECT OF TARGET FAMILIARITY}

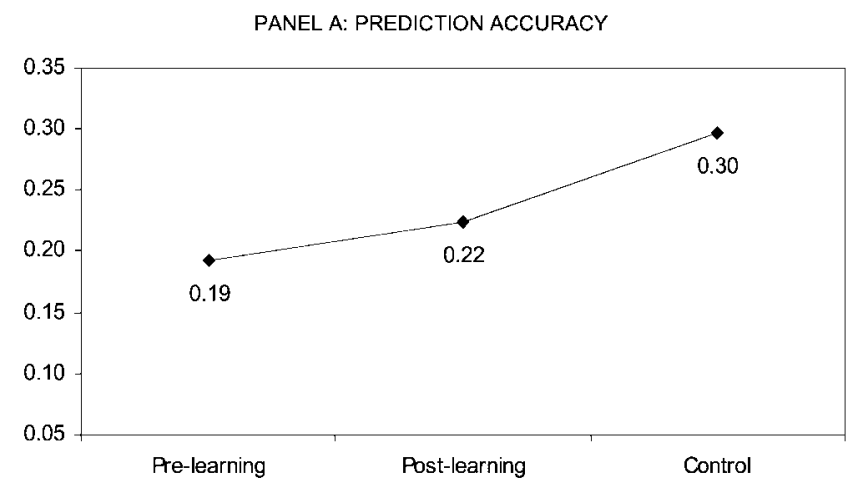

PANEL B: ACCURACY-BY-OWN ATTITUDES
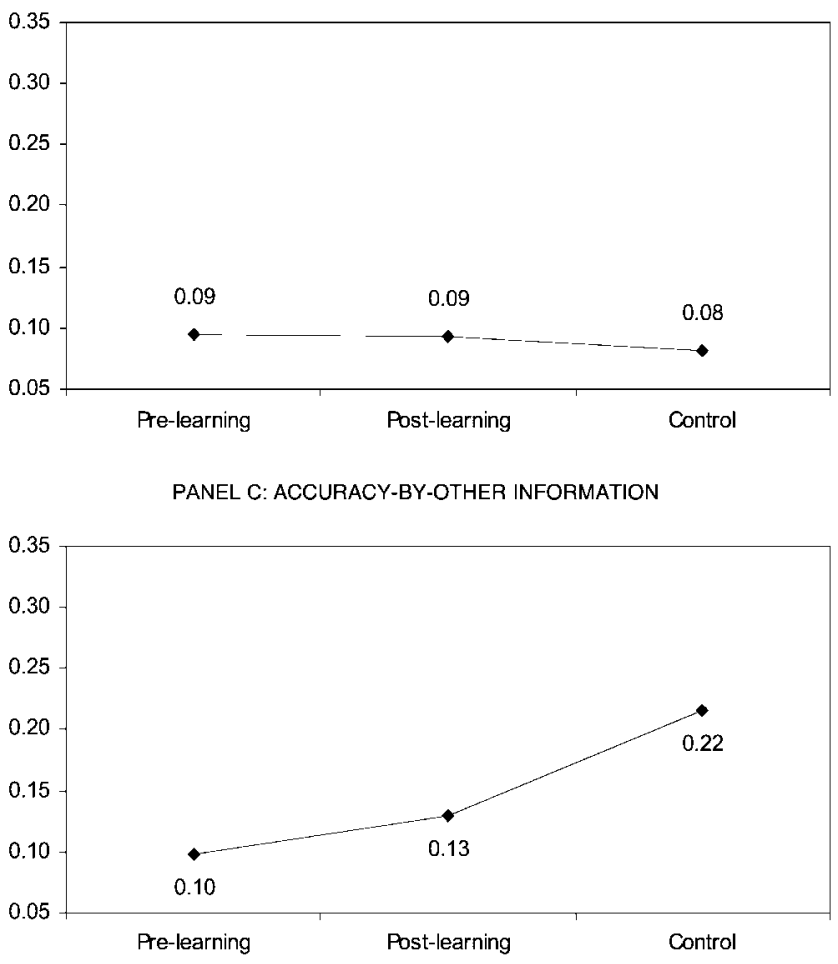

similarity had again a positive effect on accuracy $\left(M_{\text {low }}=\right.$ $\left..16, M_{\text {high }}=.32 ; F(1,214)=39.62, p<.0001\right)$.

While we observed only a positive effect of similarity $\left(M_{\text {low }}=.01, M_{\text {high }}=.17 ; F(1,214)=253.81, p<.0001\right)$ on accuracy-by-own attitudes (see panel $B$ of fig. 3 ), the effects of familiarity on accuracy-by-other information were again in favor of the retrieval explanation $(F(2,214)=$ 9.86, $p<.0001$; panel $C$ of fig. 3). The accuracy was lower for participants in the pre- and postlearning conditions $(M=.11)$ compared to those in the control condition $(M=.22 ; F(1,214)=25.63, p<.0001)$ but did not differ between the prelearning $(M=.10)$ and postlearning con- 
ditions $(M=.13 ; F(1,214)=1.43, p>.23)$. Thus, the negative effect of target familiarity on the prediction quality of other information could not be accounted for by an attention or learning bias but was mainly caused by the retrieval of less diagnostic information during the prediction task.

Consistent with the previous results on the level of projection, high similars $(M=.49)$ were found to put more weight on their own attitudes than low similars $(M=.29$; $F(1,214)=53.90, p<.0001)$. Again, target familiarity did not affect projection of own attitudes $\left(M_{\text {pre }}=.41\right.$, $\left.M_{\text {post }}=.42, M_{\text {control }}=.32 ; F(2,214)=2.04, p>.12\right)$.

\section{Discussion}

The results of experiment 3 are inconsistent with the encoding explanations but in line with a retrieval explanation. Although partners attend to and learn from feedback about each other's product attitudes, they fail to retrieve this diagnostic information when predicting their partner's attitudes toward products of the same category. It should be noted that similarity did not qualify this negative effect of target familiarity in the present experiment. Although average attitude similarity did not differ significantly from that in previous experiments $(F(1,460)=.39, p>.53)$, participants seemed to project less in the current experiment $(F(1,460)=2.80, p<.09)$. Unlike predictors in the previous experiments, participants in this experiment already received all 30 instances of attitude feedback before predicting their partner's attitudes. This could explain why the participants of this experiment relied more on other information beyond own attitudes, even when attitude similarity is high. This higher reliance on other information very likely induced the observed retrieval problems for all participants.

\section{GENERAL DISCUSSION}

Research on product attitude predictions suggests, and this research confirms, that familiarity with another consumer is not particularly helpful when predicting the other's product attitudes. Moreover, our study provides the first evidence that target familiarity even hurts prediction accuracy in the presence of product-specific attitude feedback. The negative effect of target familiarity on prediction accuracy was especially strong when predictor and target had dissimilar product attitudes. Consistent with the literature on false consensus (e.g., Marks and Miller 1987), this could indicate that consumers continue to rely heavily on their own product attitudes when predicting those of a familiar, but dissimilar, target. However, the observation that predictors reduced the weight of their own attitudes when similarity with their partner was low, thereby increasing the weight of other information, is inconsistent with this overprojection bias (experiment 1). Thus, the negative effect of target familiarity on prediction accuracy is very likely caused by differential quality of other information beyond own attitudes. One explanation that can account for this is the low validity of prestored partner information. Consistent with previous studies (e.g., Davis at al. 1986), pre-stored information about familiar targets was not more diagnostic than general and stereotypical information about strangers when predicting furniture preferences (experiment 2). However, it is important to note that pre-stored partner information was also not significantly worse than general and stereotypical information about strangers. Hence, lower quality of pre-stored partner information cannot account for the negative familiarity effect that was observed when attitude feedback was presented. Thus, although overprojection of own attitudes and low validity of pre-stored information about familiar others may have contributed to the low levels of prediction accuracy that are observed in previous studies, they cannot explain the negative familiarity effect found in the current experiments.

Instead, our results suggest that predictors put an overly heavy weight on pre-stored partner information relative to specific information about the partner's product attitudes (experiment 3). Why would consumers underweigh new and more diagnostic partner feedback? First, the predictor's ability to recall recently observed product attitudes was not affected by target familiarity. This indicates that consumers attend to feedback about their partner's product attitudes at least as well as to that of an alleged stranger. Furthermore, predictors learned equally well from feedback belonging to the partner as from feedback belonging to a stranger. Yet, consumers were still less accurate in predicting their partner's product attitudes during the subsequent prediction task. So instead of taking full advantage of the newly encoded attitude information, partners are still inclined to retrieve pre-stored target information that is less diagnostic for predicting each other's product attitudes.

Now that retrieval biases are identified as the main underlying problem in the prediction of familiar others' product attitudes, the question of why target familiarity induces these retrieval biases arises. When target familiarity is high, the few diagnostic cues from product-specific attitude feedback are among an enormous amount of pre-stored target information. As documented in the no feedback condition of experiment 2, this pre-stored information is not very diagnostic for predicting product-specific attitudes. Still, predictors are found to use irrelevant prediction cues, thereby diluting the impact of the newly encoded product-specific information (e.g., Nisbett et al.1981). Moreover, this dilution effect is found to be even stronger when predictors are accountable for their prediction (Tetlock and Boettger 1989). Presumably, predictors also felt more accountable when predicting the attitudes of familiar others, like their partner, compared to those of a complete stranger, and this may have further bolstered the dilution of newly encoded and truly diagnostic attitude information. More support for this reasoning is also found in the introspection literature. Too closely analyzing reasons for our preferences might cause less optimal prediction of our true preferences (e.g., Wilson and Schooler 1991). Similarly, reflection about why partners may like or dislike a certain product may cause predictors 
FIGURE A1

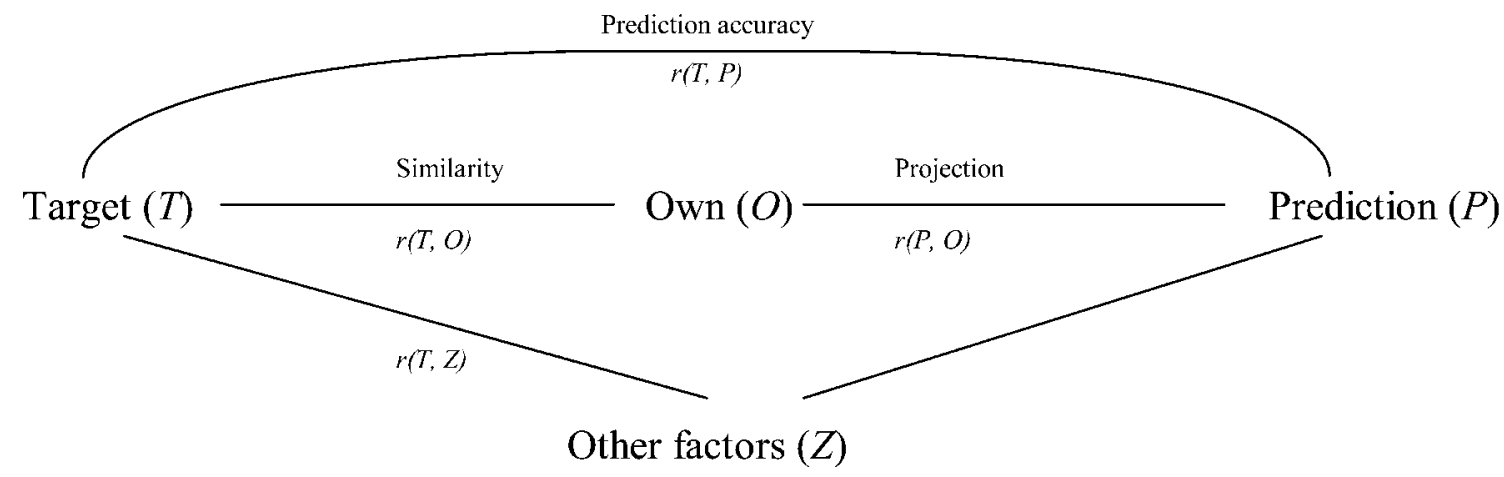

NotE.-Based on Hoch (1988, fig. on 317). Used by permission.

to retrieve less diagnostic information out of the huge amount of partner information that is available to them.

It should be noted that similarity in product attitudes was found to outweigh to a large extent the retrieval problems due to target familiarity (experiments 1 and 2). When attitude similarity was high, predictors relied heavily on their own attitudes to predict those of their partner and did not retrieve a lot of other information beyond own attitudes. Thus, retrieval biases are unlikely to emerge when attitude similarity is high because predictors simply project their own attitudes. One could argue that situations of low attitude similarity are artificial because the literature suggests that similarity is an important determinant of both partner choice and relationship success (e.g., Buss 1985). We argue that this is undoubtedly true for important aspects of life (e.g., ideology, values) but not with respect to all the innumerable specific preferences that drive decisions in everyday consumer life. Especially in these situations where similarity is low and feedback provides an important source of information, target familiarity has been found to negatively affect prediction accuracy. Moreover, when predictors possess an extended amount of product-specific attitude information about the partner and shift from own attitudes to other information, retrieval biases were found to be independent of attitude similarity (experiment 3 ).

Obviously, further research is needed to explore the generalizability of our results. Our experiments were limited to one product category: bedroom furniture. First, this product category was chosen because chances were small that participants had pre-stored knowledge of their partner's specific attitudes toward the presented bedroom sets. Therefore, they actually had to predict the other's attitudes instead of just retrieving them from memory (Hoch 1984). Second, buying decisions with respect to furniture are typically made jointly, and, consequently, they are a relevant prediction subject in a partner context (Davis and Rigaux 1974). We agree that this specific product category might have been quite unfamiliar to our participants (i.e., students). However, in reality, buying decisions that involve other consumers are also typically complex and uncommon (e.g., houses, cars, furniture, financial products) or concern products that were not perceived or discussed before (e.g., new vacation destinations). Nevertheless, the extendibility of our findings to more simple and common product categories warrants further investigation. Additionally, we restricted the attitude information to electronic exchange of overall product attitudes, devoid of physical contact or communication. This was necessary to extract the specific effect of target familiarity while keeping the target person constant. Despite this restriction, our results already suggest that different feedback formats can affect the prediction process (experiment 3 ). Attitude prediction accuracy is better when feedback is provided in a prediction setting as opposed to an observational setting, but the opposite is true for attitude recall. This indicates that the effect of different feedback settings might be quite complex and counterintuitive, making future research on this topic worthwhile.

\section{APPENDIX}

Figure A1 is Hoch's $(1988,317)$ representation of the interpersonal prediction task, where the predictor makes a prediction $(P)$ about the target's actual product attitude $(T)$.

Based on this framework, attitude predictions can be expressed in the following one-cue lens model (Hoch 1987, 224):

$$
P=B_{0}+B_{1} O+Z
$$

with $P=$ predicted product attitude of target, $B_{1}=$ weight given to own attitudes, $O=$ own attitudes, and $Z=$ other factors used to predict the target's attitudes. In standardized form this can be expressed as

$$
p=b o+\sqrt{ }\left(1-b^{2}\right) z
$$


with $b=$ projection level or $r(p, o)$. Prediction accuracy $r(p, t)$ can than be written as

$$
r(t, p)=b r(t, o)+\sqrt{ }\left(1-b^{2}\right) r(t, z)
$$

with $b r(t, o)=$ accuracy-by-own attitudes, and $\sqrt{ }(1-$ $\left.b^{2}\right) r(t, z)=$ accuracy-by-other information.

\section{REFERENCES}

Alba, Joseph W. and J. Wesley Hutchinson (2000), "Knowledge Calibration: What Consumers Know and What They Think They Know," Journal of Consumer Research, 27 (2), 123-56.

Aron, Arthur, Elaine N. Aron, Michael Tudor, and Greg Nelson (1991), "Close Relationship as Including the Other in the Self," Journal of Personality and Social Psychology, 60 (2), 241-53.

Broniarczyk, Susan M. and Joseph W. Alba (1994), "Theory versus Data in Prediction and Correlation Tasks," Organizational Behavior and Human Decision Processes, 57 (1), 117-39.

Buss, David M. (1985), "Human Mate Selection," American Scientist, 73 (January-February), 47-51.

Childers, Terry L. and Akshay R. Rao (1992), "The Influence of Familial and Peer-Based Reference Groups on Consumer Decisions," Journal of Consumer Research, 19 (2), 198-211.

Cohen, Claudia E. (1981), "Person Categories and Social Perception: Testing Some Boundaries of the Processing Effects of Prior Knowledge," Journal of Personality and Social Psychology, 40 (3), 441-52.

Davis, Harry L., Stephen J. Hoch, and E. K. Easton Ragsdale (1986), "An Anchoring and Adjustment Model of Spousal Predictions," Journal of Consumer Research, 13 (1), 25-37.

Davis, Harry L. and Benny P. Rigaux (1974), "Perception of Marital Roles in Decision Processes," Journal of Consumer Research, 1 (1), 51-62.

Davis, Jody L. and Caryl E. Rusbult (2001), “Attitude Alignment in Close Relationships," Journal of Personality and Social Psychology, 81 (1), 65-84.

Hoch, Stephen J. (1984), “Availability and Interference in Predictive Judgment," Journal of Experimental Psychology: Learning, Memory, and Cognition, 10 (4), 649-62.

- (1987), "Perceived Consensus and Predictive Accuracy: The Pros and Cons of Projection," Journal of Personality and Social Psychology, 53 (2), 221-34.

- (1988), "Who Do We Know: Predicting the Interests and Opinions of the American Consumer," Journal of Consumer Research, 15 (3), 315-24.

Kenny, David A. and Linda K. Acitelli (2001), "Accuracy and Bias in the Perception of the Partner in a Close Relationship," Journal of Personality and Social Psychology, 80 (3), 439-48.

Krishnamurthi, Lakshman (1983), "The Salience of Relevant Others and Its Effect on Individual and Joint Preferences: An
Experimental Investigation," Journal of Consumer Research, $10(1), 62-72$.

Marks, Gary and Norman Miller (1987), "Ten Years of Research on the False Consensus Effect: An Empirical and Theoretical Review," Psychological Bulletin, 102 (1), 72-90.

Menon, Geeta, Barbara Bickart, Seymour Sudman, and Johnny Blair (1995), "How Well Do You Know Your Partner? Strategies for Formulating Proxy-Reports and Their Effects on Convergence to Self-Reports," Journal of Marketing Research, 32 (1), 75-84.

Moreland, Richard L. and Robert B. Zajonc (1982), "Exposure Effects in Person Perception: Familiarity, Similarity, and Attraction," Journal of Experimental Social Psychology, 18 (5), 395-415.

Nisbett, Richard E. and Ziva Kunda (1985), "Perception of Social Distributions," Journal of Personality and Social Psychology, 48 (2), 297-311.

Nisbett, Richard E., Henry Zukier, and Ronald E. Lemley (1981), "The Dilution Effect: Nondiagnostic Information Weakens the Implications of Diagnostic Information," Cognitive Psychology, 13 (2), 248-77.

Park, C. Whan (1982), "Joint Decisions in Home Purchasing: A Muddling-Through Process," Journal of Consumer Research, 9 (2), 151-62.

Solomon, Michael R. (1986), “The Missing Link: Surrogate Consumers in the Marketing Chain," Journal of Marketing, 50 (4), 208-18.

Stapel, Diederik A. and Norbert Schwarz (1998), "Similarities and Differences between the Impact of Traits and Expectancies: What Matters Is Whether the Target Stimulus Is Ambiguous or Mixed," Journal of Experimental Social Psychology, 34 (3), 227-45.

Swann, William B. and Michael J. Gill (1997), "Confidence and Accuracy in Person Perception: Do We Know What We Think We Know about Our Relationship Partners?” Journal of Personality and Social Psychology, 73 (4), 747-57.

Tetlock, Philip E. and Richard Boettger (1989), "Accountability: A Social Magnifier of the Dilution Effect," Journal of Personality and Social Psychology, 57 (3), 388-98.

Weitz, Barton A., Harish Sujan, and Mita Sujan (1986), "Knowledge, Motivation, and Adaptive Behavior: A Framework for Improving Selling Effectiveness," Journal of Marketing, 50 (4), 174-91.

West, Patricia M. (1996), "Predicting Preferences: An Examination of Agent Learning," Journal of Consumer Research, 23 (1), 68-80.

Wilson, Timothy D. and Jonathan W. Schooler (1991), “Thinking Too Much: Introspection Can Reduce the Quality of Preferences and Decisions," Journal of Personality and Social Psychology, 60 (2), 181-92.

Wooten, David B. (2000), "Qualitative Steps toward an Expanded Model of Anxiety in Gift-Giving," Journal of Consumer Research, 27 (1), 84-95. 